\title{
Design of Intelligent E-Learning Assessment Framework Using Bayesian Belief Network
}

\author{
Rohit. B .Kaliwal ${ }^{1}$, Santosh. L. Deshpande ${ }^{2}$ \\ ${ }^{1}$ Dept. of CSE, VTU, Belagavi-590018, Karnataka, India \\ ${ }^{2}$ Dept. of CSE, VTU, Belagavi-590018, Karnataka, India \\ ${ }^{1}$ rohit.kaliwal@gmail.com \\ ${ }^{2}$ sldeshpande@gmail.com
}

\begin{abstract}
An Intelligent Tutoring Systems is a type of knowledge-based system whose main agenda is to efficiently supplement a human tutor with a machine. Dissimilar to conventional classroom teaching, Intelligent Tutoring Systems (ITSs) have the ability to fit according to the necessity of an individual learner. More emphasis has been laid on various types of e-learning systems. In this work, a probability-based ITSs system is proposed consisting of four models specifically the learner's behaviour model, pedagogical model, knowledge base model and learner assessment model. The importance has been given to the learner assessment model where an element of uncertainty has been introduced and handled by the Bayesian Belief Network (BBN). The purpose of the learner assessment model is to rightly detect the knowledge level of each learner based on their reply to the level of questions, where the level of questions is random to the process of assessment to the learner. The uncertainty factor has been defined in terms of success and failure parameters. Success is the probability that a learner of low cleverness level gives a right reply to a level of questions and is increased by a small probability of 0.07 , whereas Failure is the probability that a learner of high cleverness level gives a wrong reply to a level of questions and is reduced by a small probability of 0.04 . In this work during an assessment of the knowledge level of a learner, the system has incorporated the uncertainty factors of Success and Failure with the help of Bayes' rule and has found promising results that take into account the possibility of Success or Failure.

Keywords: Intelligent Tutoring Systems (ITSs), Assessment Framework, Knowledge Assessment, Bayesian Belief Network (BBN), E-learning
\end{abstract}

\section{Introduction}

The knowledge in education method is compound, but at the equal instance may shift the concept of education and training. Electronic Learning (E-Learning) offers a knowledge enablers and the receiving issue $[1,2]$.

\section{Corresponding Author}

Dept. of CSE, VTU, Belagavi-590018, Karnataka, India rohit.kaliwal@gmail.com

According to a twenty-year chronology of e-learning development, Rosenberg in [3] made available in a significant way chart for upholding the e-learning in sustainable and nonstop growth. So far the e-learning is a single of the leading trends in learning. In the middle of the enormous increase of COVID-19, largely learning institutions have shifted to e-learning.

E-learning is increasing at a quick speed. E-learning has been confirmed to be victorious, given the fact that several universities around the world provide certified e-learning assessment. However, a rapid move in the traditional teaching approach to e-learning delivery of the courses may encounter some challenges, especially when it comes to elearning assessment.

E-learning draws awareness of a significant move in the educational hypothesis. Owing to the complication of the developing concept, the potential random of knowledge requires the development of information like skill, knowledge free and assessment [4].

Intelligent Tutoring Systems (ITSs) also known as intelligent systems is a computer system that aims to give urgent and tailored instruction or comment to learners, typically without requiring intervention from a human tutor by a machine which provides personalized tailored instructions and feedback to the learners. The major difference between the ITSs and the traditional classroom is that ITSs can fit according to the necessity of each learner. A human tutor to cater to the needs of every learner in a classroom. Another advantage of the intelligent tutoring systems is the removal of time and space complexity of the real world unlike a regular classroom [5]. It aims to reform the online education system.

ITSs are categorized through Artificial Intelligence (AI) techniques from intelligent systems such as Machine Learning or Data Mining in constructing its mechanisms \& inside the process $[6,7]$. Even though ITSs in exercise have diverse architectures they all contribute to four basic mechanisms as shown in figure 1:

1. The Knowledge Base Model or Expert Model contains a detailed description of the user's knowledge. This model contains a superset of all concepts, strategies, rules etc. A Knowledge Base Model contains all possible steps for its solutions. It acts as a reference to assess a learner's assessment.

2. Learner's Behaviour Model contains the description of the knowledge level of the learner along with their misconceptions and knowledge gaps. It can be represented as an overlay on the individual knowledge base. A learner solves a problem activity is traced according to the knowledge base in order to exactly identify and verifying 
knowledge gain by the learner. Learner's Behaviour is the most crucial task of the ITSs.

3. The Pedagogical Model acts as a support to the learners to help the process of learning. It takes input from both Learner's Behaviour and Knowledge Base in order to provide recommendations and instructions to the learner. 4. The User Interface Model acts as an interface between the ITSs and the learner logged in.

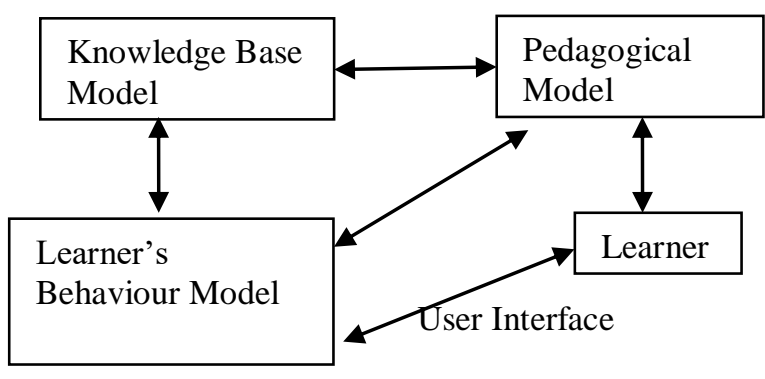

Fig.1 A Typical Intelligent Tutoring System Framework

The Main assessment followed by the existing ITS whose goal is to determine a learner's assessment about a particular domain that is how precisely and sensibly it assess a learner based on learner level of questions. Even though a learner provides a right (or wrong) reply, it cannot be exactly concluded by the system what the true state of assessment of the particular learner really is. If a learner whose past academic history is excellent and continuous assessment is good, but there are few mistakes in learner response, but learner not expected from that, there is a high possibility that the learner did a simple mistake in his/her answer. On the contrary, if a learner with low continuous performance suddenly receives excellent grades, there is a possibility of success which is a combination of random answering and adopting unfair means.

In the proposed model the possibilities of the existing system are taken into the consideration and are been added in a learner assessment model mechanism that inputs the learner current knowledge level and their response into a Bayesian Network to assess probabilities of success or failure. Then these probabilities are used to calculate the updated knowledge level of the learner.

This section is followed by the background work explaining the existing e-learning model. The section 3 explains about the proposed system of ITS followed by the Bayesian Belief Network model in section 4. The section 5 explains about learner's behaviour model followed by the learner assessment model section. The section 7 explains about the results and discussions followed by conclusion and references.

\section{Literature Review}

E-learning has been an individual of the foremost trends in learning; such significance enhances the concentration to a vital move into the learning hypothesis. Owing to the difficulty of embryonic hypothesis, the potential dynamics of knowledge needs development of information free and assessment. It tries to lay in hand a revolutionary plan of an autonomous and intelligent e-learning system. In which the machine learning and client movement investigation play the role of a regular assessor for the skill level. It is essential to judge the skill level in demand to adapt content presentation and to have a more sensible assessment of online learner [1]

In [3] e-learning it is an outstanding foundation for any institute investigating the required to execute structure and designed for individual institutes with well-known elearning programs. Scheme and equipment are obtainable realistic, echo industry methodologies to achieve range combination of e-learning into the learning institute. Elearning is well-known commerce metrics of cost, quality, service and speed are significant metrics. Crucial achievement reason for the implementation of an e-learning scheme including culture, champions, communication, and change is the reason for the most vital of a few fresh enterprise schemes.

An Intelligent Tutoring System (ITS) is a type of knowledge-based system whose main agenda is to efficiently substitute a human tutor by a machine. A probability-based ITS system is proposed consisting of four models namely student model, tutor model, domain model and a student evaluation model. The emphasis has been given on the student evaluation model where an element of uncertainty has been introduced and handled by the Bayesian network. The purpose of the student evaluation representation is to correctly detect the knowledge level of each student based on their response to questions [5].

A student knowledge modelling algorithm is a probabilistic area within an intelligent tutoring system. Diagnosing skills are assessed the actual student model based on the student answers to questions. Confirmation and updating of the model are conducted based on the identity of the students and model answers between them. Three diverse strategies namely coarse, refined, and blended are updating the knowledge to the learner. It is assessed by different assessment of the stride and indicated the output of the parametric learn $[8,9]$.

In [10] the students and teachers for the student structure are to maintain the core idea of the student model. Together with human and the system are able to advantage as of this communication. The student model has representational and communication issues arise and creation for inspection. The current ViSMod (Visualization of Bayesian Student Models) is an integrated tool to visualize and examine for distributed Bayesian student models.

In [11] the network monitoring is done manually. Usually, network administrators are responsible for efficient management and monitoring of the network. To overcome these practical problems it has move toward the Mobile Agents Based Network Monitoring (MABNM). This will give the system administrators a relief and make network monitoring a more flexible and easily manageable work. 
The familiar presence of the learner belongs to the diverse country is analyse base on top of unlike attributes such as designed actions, part academic and quantity of time they network through the topic. Therefore comparing with the learner paradigm marks of particular country $\&$ it has been done that the marks are not the simplest thing to signify the appropriate patient of the subject. The examination can be widespread to obtain into deliberation the last characteristic such as 'certified', 'explored' etc [12, $13]$.

When training systems aim to provide the student with an interactive assist, it desires to get the following information about him. The present information about the student and the outcome the learner is presently willing to accomplish. So as to, it has got to do jointly evaluation and plan gratitude. These modeling responsibilities engage a far above the ground point of improbability at what time learner is authorized to track a range of queue of logic \& be not necessary to demonstrate all their analysis clearly [14, $15,16]$.

\section{Proposed System of ITSs}

ITSs model allows a learner to gain knowledge on concept/domains a particular subject with the help of an etutor. The knowledge level and level of questions of a learner at an instant are measured with the help of learner assessment model. This model makes it possible with a prerequisite knowledge level to access questions and answers from them. Based on the responses to the question, this module provides a performance of the learner. If the learner achieves a performance above a particular threshold, it is entitled to questions of advanced knowledge level. Likewise the knowledge level is enhanced as the participant goes on solving the question in presented ITSs model. Based on the knowledge level, the learner assessment model also suggests learning documents which is accessible via the tutoring module. The underlying technology used in Bayesian Networks.

The necessity of any ITSs model to be modular is its ease to update the system when necessary. A simple change in the system does not require the need to reconstruct the whole model. This is the reason why a typical ITS is modelled into different mechanisms has proposed intelligent e-learning assessment framework shown in figure 2 .

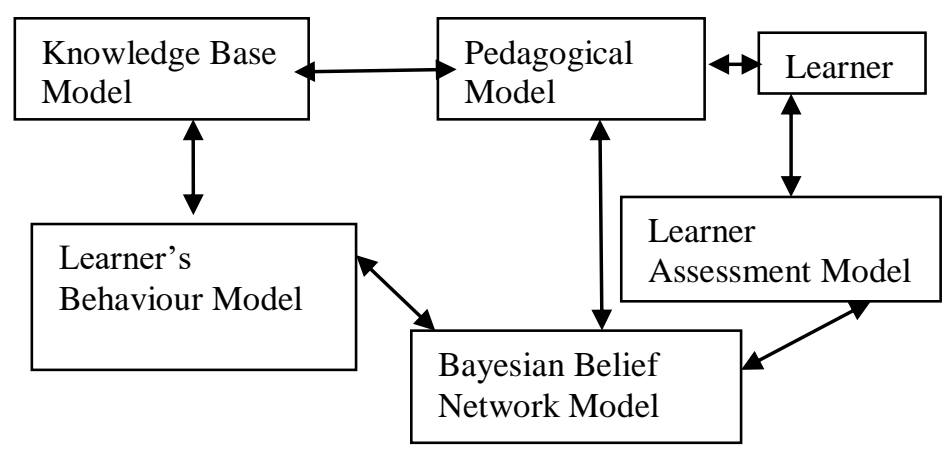

Fig.2 Proposed Intelligent E-Learning Assessment Framework

The proposed model contains detailed structure and information for each course. It consists of learning material of all the sub-topics of the course. The Knowledge Base Model is designed in the form of a tree-like structure. It is divided into all the fundamental sub-topics, which are again sub-divided. Each of the leaf nodes represents a particular concept. Corresponding to each concept there is a set of level of questions that will be used to assess learner assessment about that concept.

The Pedagogical Model acts as a support to the learners to help the process of learning. It takes input from both Learner's Behaviour and Knowledge Base to provide recommendations and instructions to the learner. It represents the instruction approach and it controls all pedagogical process.

When compared to the existing system, Knowledge Base Model and Pedagogical Model remain the same but remaining Bayesian Belief Network Model, Learner's Behaviour Model and Learner Assessment Model are been modified and explained in detail in further sections.

\section{Bayesian Belief Network Model}

Bayesian Belief Network (BBN) was originally developed as a knowledge representation with human experts. BBN (Pearl, 1988) have become accepted and used widely for reasoning under uncertainty [5]. Probabilistic causal relationships among variables are represented as a Directed Acyclic Graph (DAG). Using prior and conditional probabilities are attached to each node it is possible to propagate changes in probability values on receipt of evidence. These characteristics make BBN a powerful technique to model learner's knowledge by representing causal and probabilistic relationships among concepts and guaranteeing consistency of beliefs when new evidence (knowledge) is included in the model.

Bayesian Belief Network is a compact and theoretically sound probabilistic graphical structures which are used as a tool for building a model to represent probability distribution over a given problem domain. It is a mathematically solid and efficient mechanism to provide insights on imprecise and uncertain information. In a 
Bayesian Network, any observable state or feature is represented by a node and any interdependencies among the features are represented by directed arcs. These nodes are associated with a Conditional Probability Table (CPT) representing the probability of their occurrence conditions by their parent nodes as shown in table 2 and 3 . The proposed model is a designed network to assess the probability of failure or success for each learner answering level of questions as shown in figure 3.

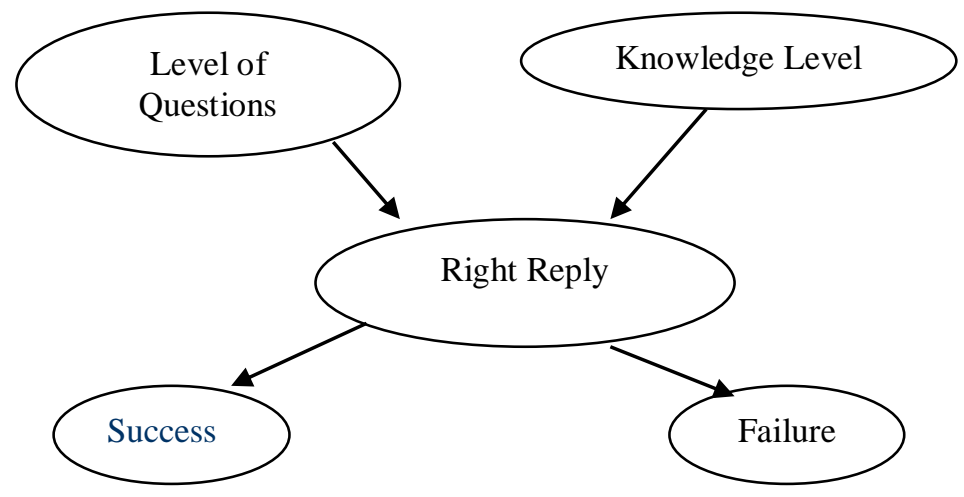

Fig.3 Assessment model construction using BBN

\section{Learner's Behaviour Model}

In the proposed system knowledge level needs to be initialized for each learner before the start of the learning process. The professor can determine the initial knowledge level of every learner for every idea from Learner's Behaviour Sequence as shown in figure 4.

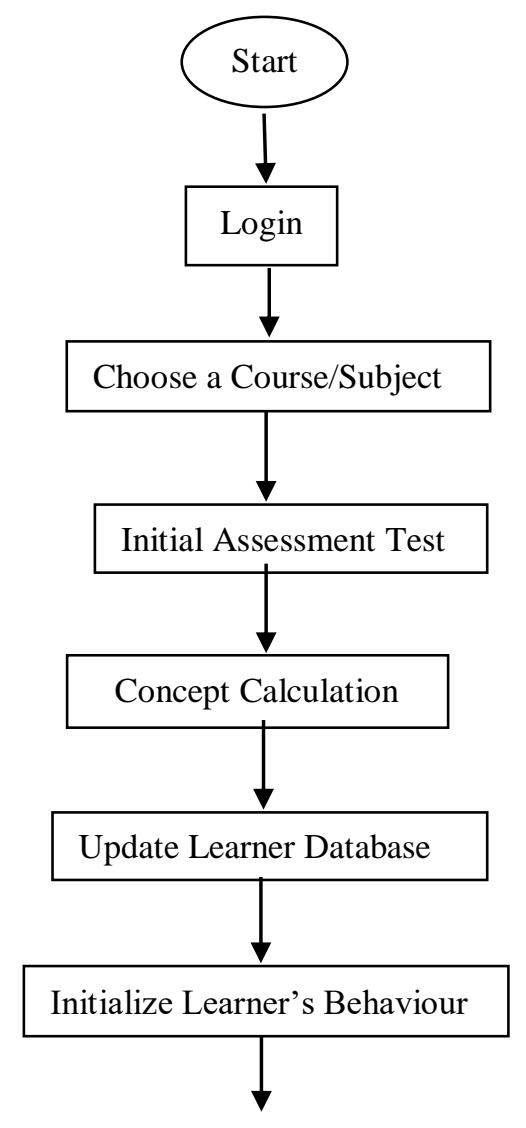

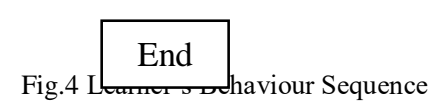

Initially, a learner logs into the system. Then he/she chooses from the available courses. A pre-evaluation test is performed which is used to determine the knowledge levels for each concept. This is used to initialize the prior learner database. The pre-assessment test consists of 50 levels of questions. Each level of questions is made up of a combination of several concepts. Here each of the concepts in the question has a certain probability associated with it. Hence the reply to each question is used to compute the probabilities for a different level of concepts. This procedure gives us a certain idea about the initial knowledge level for each learner.

Each learner will be having a definite knowledge level for each concept. Once the initial assessment is finished, all the calculated concepts are categorized into three stages as satisfactory, more satisfactory and most satisfactory. This is helpful to keep a well-defined understanding of the current knowledge level for each concept so as to provide the right guidance to the learner. The required learning documentation corresponding to each concept level is provided to the learner's by a pedagogical model so that learner can improve themselves by learning.

\subsection{Correlation of Learning and Assessment process}

Once the pre-test is over the revision procedure combines the Learner's Behaviour Model with the Knowledge Base Model to give-out suitable course content to the learner. Dissimilar kinds of adaptive knowledge are accessible. The proposed system uses a random process technique. A Random process is implemented to the current knowledge level of the learner. Random process technique is different a set of questions given to the learner from the complexity of all section domains in a course. The proposed system conceals section that has section knowledge levels lower than the learner's overall knowledge level.

\section{Learner Assessment Model}

After the learner's have completed all the learning exercises, a learner has to undergo the final assessment test which will recalculate the concept levels efficiently. Level of questions from different concepts are randomly specified to the learner's and their replies along with the previous knowledge levels are calculated using the concept of Bayesian Belief network based on the Corbett and Anderson's Bayesian Knowledge Tracing model. This is the key functionality of Learner Assessment Model.

In order to obtain information about the learner's current knowledge about a domain, the learner's replies to specified level of questions are used. But it is not necessary that a learner replies precisely only if he/she has the knowledge related to the domain. There are chances that learner can also make random success. On the other 
handset of learner having knowledge about the domain can make the wrong choice which leads to making a failure. This is known as unreliable information. So the system should consider all the evidences it has, including the learner's reply, to decide what is the current learner knowledge. This requires reasoning under uncertainty which is handled by a Bayesian Belief network where the estimate has been applied.

Compute the probability of a learner's current knowledge about a particular domain using the fundamentals of Corbett and Anderson's knowledge tracing model. From figure 3, the following are the description:

i. Here each level of questions are differentiated to a learner into a particular level of questions (LQ) (from 0-1).

ii. All the learner's said to be already graded to a certain clever stage (CS) (ranging from 0-1).

iii. In the proposed model a certain learner appearing for the question either replied rightly $(\mathrm{R})$ or wrongly.

iv. A learner with lower knowledge level is more likely to give a wrongly replied, but there is a slight chance that learner might answer rightly by making either a Success or fraud (called $\mathrm{S}$ ).

v. On the other hand a student with a higher knowledge level can also answer wrongly, by making a silly mistake or Failure (called F)

vi. Let $\mathrm{Ai}-1$ be the initial knowledge probability of a learner before answering a level of questions, either rightly or wrongly. This parameter will be dynamically updated for each reply.

vii. The learner also has a probability of learning a skill (called L), while answering a question.

Hence we compute equations to compute learner's knowledge from Bayesian Knowledge Tracing.

$\mathrm{P}(\mathrm{Ai}-1 \mid \mathrm{RN})=\mathrm{P}(\mathrm{Ai}-1)(1-\mathrm{P}(\mathrm{F})) / \mathrm{P}(\mathrm{Ai}-1)(1-\mathrm{P}(\mathrm{F}))+(1-$ $\mathrm{P}(\mathrm{Ai}-1) \mathrm{P}(\mathrm{S}))$------- Equation 1.1

$\mathrm{P}(\mathrm{Ai}-1 \mid \sim \mathrm{RN}=\mathrm{P}(\mathrm{Ai}-1) \mathrm{P}(\mathrm{F}) / \mathrm{P}(\mathrm{Ai}-1) \mathrm{P}(\mathrm{F})+(1-\mathrm{P}(\mathrm{Ai}-$ 1)(1-P(S)) )----------- Equation 1.2

As its already mentioned that the entire learner already has an initial knowledge probability which is to be reassessed and updated, taking into account the reply given to a question by the help of Bayesian rule. The updating is done as follows:

Once the output posts are received the nth interaction for the appropriate changes in the learner knowledge at an nth time can be incorporated for this, also needs to determine whether a learner is in the learned state or not. Two possible cases are taken into account for this:

$\mathrm{P}$ (Ai | Ai-1, answer)

This probability denotes being in the learned state given the student was already in the learned state.

$\mathrm{P}(\mathrm{Ai} \mid \sim \mathrm{Ai}-1$, answer $)$

This probability denotes being in the learned state given the student was not in the learned state. Adding the above two mentioned cases the probability of learning of each learner can be obtained as:

$\mathrm{P}(\mathrm{Ai} \mid$ answer $)=\mathrm{P}(\mathrm{Ai} \mid \mathrm{Ai}-1$, answer $) * \mathrm{P}(\mathrm{Ai}-1 \mid$ answer $)$ $+\mathrm{P}(\mathrm{Ai} \mid \sim \mathrm{Ai}-1$, answer $) * \mathrm{P}(\sim \mathrm{Ai}-1 \mid$ answer $)------$ -Equation 1.3
The assessment part is executed based on a graphical path as shown in figure 5: Measured a place of stage questions for a subject individual accessible by the eLearning structure. The position of questions is alienated into three stages like satisfactory stage, more satisfactory stage and most satisfactory stage. Under every position of questions, there is division. For example in a satisfactory stage, built-up questions that $\mathrm{E}=$ \{Satisfactory much, Satisfactory more, Satisfactory most $\}$. Likewise, questions are built-up for the last two stages like More Satisfactory \& Most Satisfactory. For each questions (nodes) are given has probability as shown in table 1.

a. Satisfactory Stage Questions

E1,E2,E3,.........E27 and E30 are set of Satisfactory stage questions.

$\mathrm{E} 1, \mathrm{E} 2, \mathrm{E} 3, \ldots . . \mathrm{E} 9$ and $\mathrm{E} 30$ are satisfactory questions, $\mathrm{E} 10, \mathrm{E} 11, \mathrm{E} 12 \ldots . . . \mathrm{E} 18$ are more satisfactory questions and $\mathrm{E} 19, \mathrm{E} 20, \ldots . . \mathrm{E} 22, \mathrm{E} 24, \ldots \quad \mathrm{E} 27$ are most satisfactory questions.

b. More Satisfactory Stage Questions

D1,D2,.....D18 and D20 are set of More Satisfactory stage questions.

$\mathrm{D} 1, \mathrm{D} 2, \ldots \mathrm{D} 6$ and D20 are satisfactory questions, $\mathrm{D} 7, \mathrm{D} 8, \ldots . \mathrm{D} 12$ are more satisfactory questions and D13,D14,...D18 are most satisfactory questions.

c. Most Satisfactory Stage Questions

MD1,MD2,...MD15 are set of Most Satisfactory stage questions.

MD1,MD2,...MD5 are satisfactory questions, MD6,MD7,...MD10 are more satisfactory questions and MD11,MD12,...MD15 are most satisfactory questions.

- If E1 is false there is slightly satisfactory much question $\mathrm{E} 4$ than satisfactory question $\mathrm{E} 1$ in Satisfactory stage questions as shown in figure 5 , and if E1 is True then satisfactory more question E10 in satisfactory level questions as shown in figure 5 .

- If E10 is false there is slightly satisfactory much question E11 than more satisfactory question E10 in satisfactory stage questions as shown in figure 5, and if E10 is true then more satisfactory question E19 in satisfactory stage questions as shown in figure 5. Where,

$\checkmark$ E1, E2, E3, E4, E5, E6, E7, E8, E9 and E30 are 1st stage.

$\checkmark$ E10, E11, E12, E13, E14, E15, E16, E17 and E18 are 2 nd stage.

$\checkmark$ E19, E20, E21, E22, E24, E25, E26 and E27 are 3rd stage.

$\checkmark$ D1, D2, D3, D4, D5, D6 and D20 are 4th stage.

$\checkmark$ D7, D8, D9, D10, D11 and D12 are 5th stage.

$\checkmark$ D13, D14, D15, D16, D17 and D18 are 6th stage.

$\checkmark$ MD1, MD2, MD3, MD4 and MD5 are 7th stage.

$\checkmark$ MD6, MD7, MD8, MD9 and MD10 are 8th stage.

$\checkmark$ MD11, MD12, MD13, MD14 and MD15 are 9th stage. 


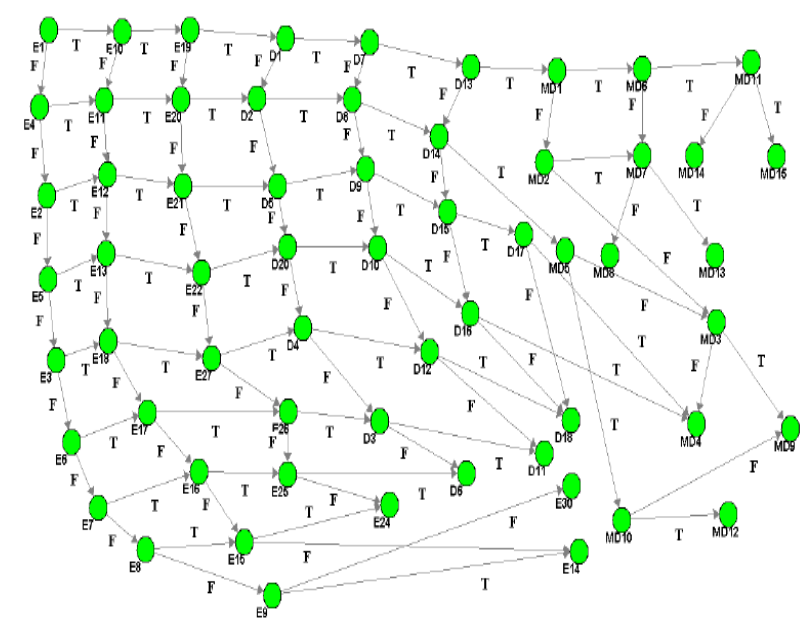

Fig.5 Construction for Level of Questions

Table 1.Conditional Probability Table

\begin{tabular}{|c|c|c|}
\hline \multirow{2}{*}{ Table 1.Conditional Probability Table } \\
\hline & PT (True) & PF (False) \\
\hline E1 & 0.5 & 0.5 \\
\hline E2 & 0.52 & 0.48 \\
\hline E3 & 0.54 & 0.46 \\
\hline E4 & 0.51 & 0.49 \\
\hline E5 & 0.53 & 0.47 \\
\hline$\cdots$ & $\cdots$ & $\cdots \cdots$ \\
\hline
\end{tabular}

From the above table 1, each question has conditional probability values. Where $\mathrm{PT}=$ Probability True and $\mathrm{PF}=$ Probability False.

\section{Results And Discussion}

During the research 20 learners were assessed; assessing each from 3 stages of a certain subject/domain from the system. Next, they were tested on levels of questions and each time their results are recorded as shown in table 2 . The set of 20 learners were closely monitored and their cleverness level was decreased by various options. The level of questions for assessment was also designed by core teachers and their opinion about the level of difficulty was directly mapped to the cleverness level which indicates the learning. This is calculated for each learner, the same is plotted in table 2 and 3.

\begin{tabular}{|c|c|c|c|}
\hline $\begin{array}{c}\text { Learner } \\
\text { USN } \\
\text { Numbers }\end{array}$ & $\begin{array}{c}\text { Knowle 2.Reply records from examine } \\
\text { level of a } \\
\text { concept }\end{array}$ & $\begin{array}{c}\text { Level of } \\
\text { Questions }\end{array}$ & Reply \\
\hline USN1 & 0.10 & High & Right \\
\hline USN 2 & 0.37 & Average & Wrong \\
\hline USN 3 & 0.23 & Low & Right \\
\hline USN 4 & 0.19 & Low & Right \\
\hline USN 5 & 0.31 & High & Wrong \\
\hline
\end{tabular}

\begin{tabular}{|c|c|c|c|}
\hline USN 6 & 0.43 & High & Wrong \\
\hline USN 7 & 0.56 & Average & Wrong \\
\hline USN 8 & 0.67 & Low & Right \\
\hline USN 9 & 0.49 & Low & Right \\
\hline USN 10 & 0.51 & High & Wrong \\
\hline USN 11 & 0.78 & High & Right \\
\hline USN 12 & 0.79 & Average & Right \\
\hline USN 13 & 0.92 & Low & Wrong \\
\hline USN 14 & 0.70 & Average & Right \\
\hline USN 15 & 0.76 & High & Wrong \\
\hline USN 16 & 0.13 & High & Wrong \\
\hline USN 17 & 0.89 & High & Right \\
\hline USN 18 & 0.76 & Average & Right \\
\hline USN 19 & 0.17 & Low & Wrong \\
\hline USN 20 & 0.45 & Average & Right \\
\hline
\end{tabular}

\subsection{Consider Cases from the above table 2}

Case1: USN1, the learner is of low cleverness level and he answered the high-level questions which he answers rightly. So there is a chance that he might have either succeed the answer or have taken some unfair means to do so. In case of a traditional case wrongly a high mark is assigned to this learner. But in this system, some probability is incorporated of success with the help of a Bayesian network to impute a few amounts of improvement.

Case2: USN 13, the learner is of high cleverness level and he answered the low-level questions which he answers wrongly. So there is a chance that he might have either made a childish mistake or have wrongly imputed the answer. In case of a traditional wrongly assign low marks to this learner. But in this system some probability of Failure with the help of a Bayesian network to a few amount of improvement.

If the learner answers only the satisfactory stage of questions, then the Cleverness Level is low (1), whereas the learner answers satisfactory stage and more satisfactory stage of questions, then the Cleverness Level is Average (2) and the learner answers satisfactory stage, more satisfactory stage and most satisfactory stage of questions, then the Cleverness Level is High (3) as shown in figure 5.

The computation for the above two cases have been shown in the following sections:

Computation 1: Learner knowledge for learner's with USN 1 the Low cleverness level who is specified a level of questions, the learner is ended with most satisfactory stage, that learner answers it rightly, where $\mathrm{P}(\mathrm{F})=0$ and $\mathrm{P}(\mathrm{S})=0.88$ from table 3:

$$
\mathrm{P}(\mathrm{Ai}-1 \mid \mathrm{RN})=\mathrm{P}(\mathrm{Ai}-1)(1-\mathrm{P}(\mathrm{F})) / \mathrm{P}(\mathrm{Ai}-1)(1-\mathrm{P}(\mathrm{F}))+(1-
$$

$\mathrm{P}(\mathrm{Ai}-1) \mathrm{P}(\mathrm{S}))$

$$
\begin{aligned}
& =0.15 / 0.15+0.868 \\
& =0.14(\text { Low Cleverness Level })
\end{aligned}
$$

In a traditional system, this learner will be graded high marks without taking into any account of whether the learner has performed any Success or unfair mean.

Computation 2: Learner knowledge for learners with USN 13 with High Cleverness level who is specified a level of questions, the learner is ended with satisfactory stage, that learner answers it wrongly, where $\mathrm{P}(\mathrm{F})=0.89$ and $\mathrm{P}(\mathrm{S})=0$ from table 4 : 
$\mathrm{P}(\mathrm{Ai}-1 \mid \sim \mathrm{RN}=\mathrm{P}(\mathrm{Ai}-1) \mathrm{P}(\mathrm{F}) / \mathrm{P}(\mathrm{Ai}-1) \mathrm{P}(\mathrm{F})+(1-\mathrm{P}(\mathrm{Ai}-$ 1)(1-P(S)))

$$
\begin{aligned}
& =0.92 * 0.89 / 0.92 * 0.89+0.08 \\
& =0.91(\text { High Cleverness Level) }
\end{aligned}
$$

In a traditional system, this learner will be graded low marks without taking into any account of whether the learner has performed any failure or silly mistake.

Table.3. Conditional Probability Table for Success

\begin{tabular}{|c|c|c|c|}
\hline \multicolumn{4}{|c|}{ Parameter } \\
\hline $\begin{array}{c}\text { Level of } \\
\text { Questions }\end{array}$ & $\begin{array}{c}\text { Cleverness } \\
\text { Level }\end{array}$ & $\begin{array}{l}\text { Right } \\
\text { Reply }\end{array}$ & $\begin{array}{c}\text { Probability } \\
(\mathbf{P}(\mathbf{S}=\mathbf{T} \mid \mathbf{C S}, \mathbf{R}, \mathbf{L Q})\end{array}$ \\
\hline High & 1 & True & 0.88 \\
\hline High & 1 & False & - \\
\hline High & 2 & True & 0.76 \\
\hline High & 2 & False & - \\
\hline High & 3 & True & 0.35 \\
\hline High & 3 & False & - \\
\hline High & 3 & True & 0.25 \\
\hline High & 3 & False & - \\
\hline Low & 1 & True & 0.43 \\
\hline Low & 1 & False & - \\
\hline Low & 2 & True & 0.23 \\
\hline Low & 2 & False & - \\
\hline Low & 3 & True & 0.12 \\
\hline Low & 3 & False & - \\
\hline Low & 3 & True & 0.66 \\
\hline Low & 3 & False & - \\
\hline Average & 1 & True & 0.73 \\
\hline Average & 1 & False & - \\
\hline Average & 2 & True & 0.67 \\
\hline Average & 2 & False & - \\
\hline
\end{tabular}
Parameter

Table.4. Conditional Probability Table for Failure Parameter

\begin{tabular}{|c|c|c|c|}
\hline $\begin{array}{c}\text { Level of } \\
\text { Questions }\end{array}$ & $\begin{array}{c}\text { Cleverness } \\
\text { Level }\end{array}$ & $\begin{array}{c}\text { Right } \\
\text { Reply }\end{array}$ & $\begin{array}{c}\text { Probability } \\
(\mathbf{P}(\mathbf{F}=\mathbf{T} \mid \mathbf{C S}, \mathbf{R}, \mathbf{L Q})\end{array}$ \\
\hline High & 1 & True & - \\
\hline High & 1 & False & 0.08 \\
\hline High & 2 & True & - \\
\hline High & 2 & False & 0.05 \\
\hline High & 3 & True & - \\
\hline High & 3 & False & 0.22 \\
\hline High & 3 & True & - \\
\hline High & 3 & False & 0.17 \\
\hline Low & 1 & True & - \\
\hline Low & 1 & False & 0.03 \\
\hline Low & 2 & True & - \\
\hline
\end{tabular}

\begin{tabular}{|c|c|c|c|}
\hline Low & 2 & False & 0.76 \\
\hline Low & 3 & True & - \\
\hline Low & $\mathbf{3}$ & False & $\mathbf{0 . 8 9}$ \\
\hline Low & 3 & True & - \\
\hline Low & 3 & False & 0.36 \\
\hline Average & 1 & True & - \\
\hline Average & 1 & False & 0.23 \\
\hline Average & 2 & True & - \\
\hline Average & 2 & False & 0.43 \\
\hline
\end{tabular}

\section{Conclusion}

In this article, proposed ITSs is capable of enhancing the traditional e-learning system by incorporating the framework using Bayesian Belief Network. Unlike traditional assessment system, where the contrast between an excellent learner and poor learner is based on the marks obtained merely is not a progressive method. Enhance the diffusion is created by considering the uncertainty. This method doesn't crisply mention that for choosing the wrong option by the learner is not penalized. Hence this research work results illustrate that assessment of the current knowledge level of each learner is corrected by incorporating both the success and failure parameter in the Bayesian network. In case of a high cleverness learner performing a failure, their knowledge level is reduced by a small probability of 0.04 . Also in case of a low cleverness learner performing a success, their knowledge level is increased by a small probability of 0.07 . Also, it's felt that in the present COVID-19 pandemic this type of system shall enhance the E-learning process.

\section{References}

1. Nazeeh Ghatasheh, "Knowledge Level Assessment in eLearning Systems Using Machine Learning and User Activity Analysis",International Journal of Advanced Computer Science and Applications, Vol. 6, No. 4, 2015.

2. Mahbobe Bani Asad Askari, Mostafa Ghazizadeh Ahsaee, "Bayesian network structure learning based on cuckoo search algorithm", 6 ${ }^{\text {th }}$ Iranian Joint Congress on Fuzzy and Intelligent Systems, 2018.

3. M. J. Rosenberg, "E-Learning: Strategies for Delivering Knowledge in the Digital Age", New York, NY, USA: McGraw-Hill, Inc., 2002.

4. Rohit B Kaliwal, Santosh L Deshpande, "Efficiency of Probabilistic Network Model for Assessment in E-Learning System", International Journal of Recent Technology and Engineering (IJRTE), ISSN: 2277-3878 (Online), Volume9 Issue-3, September 2020. Page No: 562-566.

5. Baisakhi Chakraborty and Meghamala Sinha, "Student Evaluation Model Using Bayesian Network In An Intelligent E-Learning System", IIOAB Journal, 2016, Vol. 7, ISSN: 0976-3104.

6. Oktariani Nurul Pratiwi, Yenie Syukriyah, "Question Classification for e-Learning Using Machine Learning Approach", International Conference on ICT for Smart Society (ICISS), 2020. 
7. D Baker, Ryan SJ, Albert T Corbett, and Vincent Aleven, "More accurate student modeling through contextual estimation of slip and guess probabilities in bayesian knowledge tracing", Intelligent Tutoring Systems. Springer Berlin Heidelberg, 2008.

8. Nabila Khodeir, Nayer Wanas and Nadia Hegazy, "Bayesian Based Student Knowledge Modeling In Intelligent Tutoring Systems", $6^{\text {th }}$ IEEE International Conference on E-Learning in Industrial Electronics (ICELIE), 2012.

9. Anderson $\mathrm{H}$, Koedinger M, "Intelligent tutoring goes to school in the Big City", International Journal of Artificial Intelligence in Education, pp. 30-43, 1997.

10. Juan-Diego Zapata-Rivera, Jim E. Greer, "Interacting with Inspectable Bayesian Student Models", International Journal of Artificial Intelligence in Education, 2004.

11. Rohit. B. K, "Mobile Agents Based Network Monitoring", National Conference on RIICTEM-2015, PG Centre, VTU, Kalaburagi, 2015.

12. Sonali Shankar, Bishal Dey Sarkar, Sai Sabitha, Deepti Mehrotra, "Performance Analysis of Student Learning Metric using K-Mean Clustering Approach", $6^{\text {th }}$ International Conference Cloud System and Big Data Engineering, 2016.

13. Abdulghani Ali Al-Hattami, "E-Assessment of Students Performance During the E-Teaching and Learning", International Journal of Advanced Science and Technology, ISSN: 2005-4238, Vol. 29, No. 8, pp. 1537-1547, 2020.

14. Conati C., Gertner A., Vanlehn K, "Using Bayesian networks to manage uncertainty in student modelling", Journal of User Modeling and User-Adapted Interaction, 12(4), 371-417, 2002.

15. JM Agosta E Millan and JL Perez de la Cruz, "Bayesian student modelling and the problem of parameter specification", British Journal of Educational Technology, 2001.

16. Cristina Conati and Kurt VanLehn Pola,"A student modeling framework for probabilistic on-line assessment of problem solving performance", Proceedings of the $5^{\text {th }}$ International Conference on User Modeling, 1996. 